\title{
Parents, familles et école dans un système éducatif néo-libéral
}

Réflexions sur les attentes, les inégalités et les tensions dans le contexte anglais

Parents, families and school in a neo-liberal education system. Reflections on the expectations, inequalities and tensions in the English context

Padres, familias y escuela en un sistema educativo neo-liberal

Reflexiones sobre las expectativas, las desigualdades y las tensiones en el contexto inglés

\section{Gill Crozier}

Traducteur : Jérôme Quintana

\section{OpenEdition Journals}

Édition électronique

URL : https://journals.openedition.org/ries/3108

DOI : 10.4000/ries.3108

ISSN : 2261-4265

Éditeur

France Education international

Édition imprimée

Date de publication : 1 avril 2013

Pagination : $59-68$

ISBN : 978-2-85420-599-2

ISSN : $1254-4590$

Référence électronique

Gill Crozier, "Parents, familles et école dans un système éducatif néo-libéral », Revue internationale d'éducation de Sèvres [En ligne], 62 | avril 2013, mis en ligne le 01 avril 2015, consulté le 06 juillet 2021. URL : http://journals.openedition.org/ries/3108; DOI : https://doi.org/10.4000/ries.3108 


\section{Parents, familles et école dans un système éducatif néo-libéral}

\section{Réflexions sur les attentes, les inégalités et les tensions dans le contexte anglais*}

\section{Gill Crozier}

On s'accorde généralement à dire que le néo-libéralisme en GrandeBretagne est né au début des années 1980, avec l'arrivée au pouvoir du Premier ministre Margaret Thatcher. Si diverses politiques néo-libérales ont été introduites ponctuellement en Angleterre durant cette décennie, c'est la Loi sur la réforme de l'éducation (Education Reform Act) de 1988 qui a marqué le début de la transformation la plus profonde et la plus radicale du système éducatif anglais jamais menée dans le cadre d'une politique néo-libérale. À l'époque, et aujourd'hui encore, l'intention était de permettre au marché d'améliorer le niveau et les performances scolaires, en donnant aux parents la possibilité de choisir l'établissement de leurs enfants, mais également en demandant aux enseignants et aux établissements scolaires de rendre des comptes. De fait, les politiques néo-libérales ont induit un certain nombre de comportements parentaux où l'on constate que "l'implication des parents" dépasse le simple fait d'écouter les enfants lire ou de vérifier les devoirs scolaires. Les parents jouent aujourd'hui un rôle essentiel dans leur capacité à rendre le marché de l'éducation opérationnel. En Angleterre, les parents s'attendent maintenant à pouvoir choisir un établissement pour leurs enfants et à avoir le droit de remettre en question les processus éducatifs en place, ainsi que la façon dont l'enseignement est dispensé à l'école. Les parents espèrent que ce type d'implication est utile et permet d'obtenir les résultats attendus. Néanmoins, ces attentes sont souvent contrariées.

S'investir dans le marché de l'éducation, que ce soit dans le choix d'un établissement scolaire ou dans le fait de soutenir un enfant dans ses progrès scolaires, exige des parents qu'ils aient une bonne connaissance du système éducatif et de la façon dont le marché fonctionne. Mais les parents veulent savoir aussi en quoi consiste exactement le processus éducatif susceptible d'aider leurs enfants à progresser. Les questions de classe sociale et d'appartenance ethnique jouent notamment un rôle très important dans l'investissement des parents à l'école. Ces deux identités sociales permettent d'établir des différences, et des différenciations, dans les rapports avec l'école et les degrés d'investissement. Dans les années 1990, Phillip Brown (1990) a avancé l'idée que le système éducatif

*Article traduit par Jérôme Quintana. 
britannique entrait dans une «troisième vague » de développement, caractérisée par la montée de la "parentocratie ». Selon lui, dans cette "parentocratie », la scolarité d'un enfant dépend des ressources financières et des souhaits de ses parents. Il a par ailleurs prédit avec raison que la parentocratie et la troisième vague de politiques éducatives aboutiraient à accroître les inégalités, plutôt qu'à les remettre en question.

Dans cet article, nous abordons le rôle et les attentes des parents dans un système éducatif néo-libéral. Nous proposons une réflexion sur l'impact que le marché de l'éducation a pu avoir sur ces attentes et sur les différents types d'implication des parents, en nous concentrant sur les conséquences et les implications relatives aux inégalités structurelles qui existent dans ce domaine ultra compétitif.

\section{LE MARCHÉ DE L'ÉDUCATION : INÉGALITÉS, RÉSULTATS ET ATTENTES DES PARENTS}

Les politiques et les pratiques en matière d'implication des parents proviennent souvent des États-Unis et se sont répandues aujourd'hui dans la plupart des pays d'Europe. Si certaines critiques ont pu être formulées à l'égard de ces politiques, dans une tentative de déconstruction des valeurs hégémoniques blanches qui les sous-tendent, l'implication des parents est présentée le plus souvent comme un bien en soi, ne posant pas de problème. Nous avançons néanmoins l'idée que l'implication des parents ne relève pas d'un concept uniforme, à visée unidimensionnelle, mais évolue au contraire avec le temps, à mesure que les impératifs politiques et les contextes socio-économiques évoluent eux aussi. C'est le rapport Plowden qui a pour la première fois, en 1967, émis l'idée d'une implication des parents au Royaume-Uni. On a alors encouragé les parents à améliorer le travail des enseignants en consolidant les apprentissages scolaires à la maison. Depuis, l'implication des parents a pris deux voies différentes : d'un côté, on incite les parents à agir davantage comme consommateurs et à jouer un rôle clé dans leur capacité à rendre opérationnelle la politique de marchandisation néo-libérale de l'éducation. De l'autre, on fait en sorte que l'implication des parents serve à contrôler les parents eux-mêmes, notamment les parents que l'on estime d'un « autre » milieu : celui de la communauté noire, des minorités ethniques et des classes populaires blanches.

\section{LES PARENTS COMME CONSOMMATEURS}

On a incité les parents à s'investir dans le choix d'un établissement pour leurs enfants, en leur faisant croire qu'ils avaient réellement le choix et qu'en exerçant ce choix, ils pouvaient améliorer la scolarité de leurs enfants. La réalité est quelque peu différente. Dans de nombreux cas, lorsqu'ils veulent 
envoyer leurs enfants dans l'école primaire ou l'établissement secondaire de leur secteur qui affiche apparemment d'excellents résultats, ils découvrent qu'il n'y a pas suffisamment de places. Dans leur enquête menée dans le quartier populaire de l'est de Londres, East End, Butler et Hamnett (2012) ont montré que les autorités locales chargées de l'éducation étaient incapables de garantir un enseignement de qualité, si bien que le choix des parents devenait souvent un concept dénué de sens. Les parents se sentaient contraints de chercher des établissements scolaires en dehors du secteur, et s'ils en avaient les moyens, ils changeaient de domicile et de quartier. Cette enquête a également montré que le processus de choix d'un établissement était préjudiciable aux établissements urbains de ce secteur, car ceux-ci étaient classés parmi les "mauvais ", contrairement aux autres, situés ailleurs et jugés «bons ». Les " mauvais » établissements perdaient ainsi leurs élèves, tandis que les «bons " établissements étaient en sureffectifs. Les parents se faisaient une idée du bon établissement, gage de réussite, en se fondant sur les critères étroits de la politique du "choix ", c'est-à-dire qu'en termes de réussite aux examens, ils consultaient le palmarès des établissements, les school league tables. Alors que dans de nombreux cas, les établissements en zone urbaine défavorisée avaient enregistré de très bons résultats en termes de progrès réalisés (ce que l'on appelle dans le discours politique la «valeur ajoutée »), les parents n'en tenaient pas compte dans leurs recherches.

Avoir le choix d'un établissement, demander des comptes aux enseignants, avoir voix au chapitre dans la gestion de l'établissement (par exemple en étant élu au conseil d'administration), voilà autant de droits ou de prérogatives qui concernent les parents de la classe moyenne blanche. Quand bien même les parents de la communauté noire, ou ceux issus des minorités ethniques ou des milieux populaires voudraient également s'investir dans leur établissement par ces moyens-là, nous savons grâce à de nombreuses recherches que ces groupes ne possèdent généralement ni le capital culturel nécessaire à la connaissance du monde éducatif ni le capital social requis pour le faire. Ils sont par ailleurs moins susceptibles de pouvoir faire des choix pertinents en matière d'établissement ou de pouvoir se battre pour obtenir la place voulue. Ils n'ont pas non plus les ressources financières leur permettant de déménager au cas où ils souhaiteraient s'installer dans un secteur qui compte un établissement plus attirant.

La faiblesse des performances des enfants de la communauté noire, de ceux issus des minorités ethniques et des milieux populaires demeure préoccupante en Angleterre. La politique du choix de l'établissement est présentée comme étant un mécanisme visant à offrir les meilleures chances possibles aux enfants et à leur éviter l'échec. Il n'est donc pas surprenant que les classes moyennes émergentes de la communauté noire et celles issues des minorités ethniques en Angleterre se soient investies dans le marché éducatif de la même façon que les classes moyennes blanches (Archer, 2010), afin de garantir la réussite scolaire de leurs enfants. Les recherches prouvent néanmoins que, malgré la 
mobilité sociale, les préjugés raciaux continuent d'entraver le chemin de ces communautés ethniques, que ce soit pour obtenir une place dans l'établissement voulu ou pour se faire entendre au sein de l'établissement, lorsqu'ils remettent en cause la façon de faire de l'enseignant ou la manière dont on a traité leur enfant.

\section{L'IMPLICATION DES PARENTS COMME FORME DE « POUVOIR DISCIPLINAIRE ${ }^{1}$}

En lien avec les discours sur l'implication des parents, le concept de «citoyen actif » est repris depuis longtemps par le monde politique, et incarné notamment dans l'appel lancé par le Premier ministre Tony Blair, souhaitant qu'il n'y ait "pas de droits sans responsabilités ", ce qui, dans le contexte actuel de la coalition gouvernementale dirigée par David Cameron, pourrait constituer un aspect de la "Grande Société "² (The Big Society). Selon Levitas (1998), le rôle du citoyen actif est fondamental dans une société participative. En ce sens, l'implication des parents repose probablement sur la nécessité de faire en sorte que les communautés soient reliées entre elles à "des fins communes ». Il faut noter l'importance cruciale de la base servant à définir cette finalité commune, et servant ou pouvant servir à identifier les actions entreprises, en lien avec elle. Le bon fonctionnement des établissements scolaires repose sur une certaine harmonie, une vision et des valeurs unifiées. Lorsque les parents qui siègent au conseil d'administration d'un établissement sont issus de la diversité ethnique et sociale, cela pose un problème pour cette harmonie d'ensemble, notamment lorsque ces parents sont perçus comme possédant une vision et des valeurs différentes de la norme hégémonique. Ces différences s'incarnent dans les familles des classes populaires blanches ainsi que dans les familles de la communauté noire et celles issues des minorités ethniques.

La participation des parents repose donc sur un type particulier de comportement ou de parent, que Vincent (1996) qualifie de «bon parent ». Les recherches ont montré que les enseignants ont une vision claire, mais néanmoins limitée, du "bon parent» (Vincent, 1996). La politique appliquée dans ce domaine est la suivante : lorsque les parents ne sont pas «bons", on les rappelle à l'ordre par exemple par le biais de " cours d'éducation parentale », ou en faisant appel à des mesures plus strictes, telles que des ordonnances de justice.

\footnotetext{
1. Foucault (1977) : pour parvenir à un système d'autorégulation des comportements, plutôt qu'à un système ouvertement punitif.

2. "The Big Society " était l'idée politique phare du programme électoral du Parti conservateur britannique lors des élections de 2010. Elle fait désormais partie du programme législatif de l'accord de coalition entre les conservateurs et les démocrates libéraux. Le but affiché est de créer un climat qui donne des moyens d'action aux communautés locales et aux personnes, créant une « grande société » qui reprendra le pouvoir accaparé par les hommes politiques et le rendra aux citoyens. ( $N d T$ )
} 
Certains parents ont même été condamnés à des peines de prison parce que leurs enfants étaient systématiquement en retard à l'école ou s'absentaient de façon chronique. Si la définition $\mathrm{du}$ «bon parent » demeure imprécise, il est sous-entendu que le "bon parent» est issu de la classe moyenne blanche (Crozier, 2000). Incontestablement, les parents qui, dans l'ensemble, parviennent le mieux à obtenir ce qu'ils veulent pour la scolarité de leurs enfants sont ceux issus de la classe moyenne blanche. Ceux-là ont le pouvoir d'intervenir de manière active et d'obtenir gain de cause (Crozier, 2000).

Cependant, même si les enseignants de la classe moyenne blanche identifient les parents de la classe moyenne blanche comme étant «identiques à eux ", partageant en apparence les mêmes valeurs éducatives, ils peuvent également voir en eux une menace, une gêne dans leur travail. Les parents transgressent effectivement la frontière entre profanes et professionnels (Vincent, 1996) et remettent en cause le professionnalisme des enseignants, auxquels ils demandent des comptes. Même s'il n'est pas possible d'approfondir ici la réflexion sur ce phénomène, il est néanmoins important de dire que les enseignants sont eux-mêmes mis en difficulté par les politiques néo-libérales : ils se retrouvent en effet coincés entre, d'une part, leurs propres attentes professionnelles et, d'autre part, des impératifs politiques qui changent fréquemment et la nécessité de défendre leurs pratiques face à des parents qui ont aujourd'hui du pouvoir (voir Vincent, 1996 ; Crozier, 2000).

Les recherches effectuées nous permettent de constater que les parents de la communauté noire et ceux issus des minorités ethniques ne sont généralement pas perçus comme étant "bons». Elles montrent un certain nombre de perceptions négatives et stéréotypées à l'égard des parents issues des minorités ethniques, de la part des enseignants. Cecile Wright (1992) a par exemple rapporté le fait que les parents afro-caribéens étaient considérés comme étant hostiles à l'école, comme posant problème, ayant "tendance à être (hyper) anxieux au sujet des progrès de leurs enfants ». D’autres chercheurs ont indiqué que les enseignants voyaient les parents de la communauté noire et ceux issus des minorités ethniques comme ayant des attentes irréalistes pour leurs enfants ou ne manifestant pas d'intérêt pour leur scolarité (Crozier et Davies, 2007).

Comparativement à l'idéal du «bon parent », les comportements des parents issus des minorités ethniques, tels que nous les avons décrits plus haut, induisent voire renforcent l'idée de différence, l'image de "l'autre ». Iris Marion Young (1990) interroge les comportements du groupe dominant en termes d'impérialisme culturel, qu'elle décrit comme étant " la généralisation de l'expérience et de la culture du groupe dominant, devenues la norme ». Elle avance l'idée qu'à travers ce processus, les valeurs et les expériences de ce groupe, sa façon de voir et d'interpréter les événements, se projettent sur l'ensemble de la société. Les attitudes et les comportements ainsi que les autres paramètres se mesurent à l'aune de cette norme. Ainsi, lorsque le groupe dominant se voit 
remis en cause par un autre groupe, poursuit Young, le groupe dominant se défend en affirmant sa supériorité. Et l'autre groupe est de ce fait perçu comme étant déviant, inférieur et donc désigné comme étant cet " autre ». Young affirme également que si, d'un côté, le groupe dominé est perçu de manière stéréotypée comme agissant de façon déviante/étrange et/ou inacceptable, de l'autre il devient invisible. Les possibilités offertes aux parents de la communauté noire ou à ceux issus des minorités ethniques d'intervenir en faveur de leurs enfants et d'obtenir gain de cause, semblent ainsi limitées, vu la position dans laquelle ils sont mis.

\section{LA « DIFFICULTÉ D'ACCÈS " DES ÉTABLISSEMENTS SCOLAIRES}

L'expression "difficile d'accès » s'applique d'ordinaire aux parents jugés en marge de l'école, voire de la société au sens large, donc socialement exclus, qu'il faut semble-t-il " ramener dans le giron » de l'école et rendre à nouveau acteurs (Crozier et Davies, 2007). C'est souvent ainsi qu'on se réfère aux parents des classes populaires blanches ou à ceux issus des minorités ethniques. Crozier et Davies (2007) ont toutefois démontré dans leurs travaux sur les rapports entre l'école et les familles originaires du sud-est asiatique que, le plus souvent, il est surtout difficile de s'investir dans les établissements scolaires pour les parents. Comme nous l'avons indiqué précédemment, les parents issus de ces milieux sont marginalisés, rendus invisibles et les enseignants manifestent des attentes limitées à leur égard. Les parents qui éprouvent beaucoup de mal à entrer en contact avec l'établissement et les enseignants, qui se sentent intimidés, voient leurs difficultés exacerbées par le manque de compréhension et d'écoute de la part de l'établissement. Les établissements scolaires ont tendance à traiter les parents comme un groupe homogène et leur politique en matière d'implication des parents est identique quelle que soit la situation. Souvent, ils ne tiennent pas compte de la diversité des besoins exprimés par les parents, par exemple en matière de soutien linguistique ou pour renforcer la confiance des élèves. Certains parents de milieux populaires n'ont pas vécu leur scolarité de manière positive, d'autres issus de minorités ethniques ne connaissent pas bien le système éducatif britannique. Ces deux facteurs ébranlent la confiance des parents, lorsqu'il s'agit de prendre contact avec l'établissement ou de remettre en cause les enseignants dans leur pratique. S'il arrive que ces derniers prennent conscience de cela, ils le voient alors comme une faille et non comme un problème qui demande des mesures pratiques et constructives, afin de remédier aux réticences des parents ou à leur incapacité à se rendre aux réunions parce qu'ils sont retenus par leur travail.

Les jeunes, que l'on omet souvent de prendre en compte dans les discours sur l'implication des parents, ont en réalité leur propre avis sur la question. Plusieurs études (voir notamment Reay et Ball, 1998) révèlent des différences de classes sociales dans le processus de choix d'un établissement, 
les parents de milieux populaires étant davantage susceptibles de laisser leurs enfants libres de faire ce choix, soulignant ainsi l'importance des facteurs locaux et des relations amicales qu'entretiennent les jeunes. En revanche, les parents des classes moyennes ont plus de chances d'imposer leurs souhaits, confirmant ainsi, dans l'ensemble, la thèse de la reproduction sociale et le poids de leur investissement dans la scolarité de leurs enfants. L'âge des enfants est un indicateur du degré d'implication parentale qu'ils souhaitent: les adolescents préfèrent se sentir soutenus par leurs parents, plutôt que de les voir s'investir directement dans leur scolarité. Néanmoins, en ce qui concerne la question de la diversité, de la différence et de l'image de "l'autre », Crozier et Davies (2007) sont parvenus à la conclusion que les jeunes originaires du sud-est asiatique servent de "gardiens » de la frontière qui sépare la maison de l'école, afin de se protéger des pressions exercées par les parents, mais aussi de protéger leurs parents de ce qu'eux-mêmes perçoivent comme pouvant être des propos gênants ou offensants, voire des injures racistes, en cas de convocation à une réunion dans l'établissement.

\section{LES RÉACTIONS DES PARENTS FACE À LA POLITIQUE NÉO-LIBÉRALE}

Le fait que les parents aient été poussés à devenir clients, qu'ils aient le choix de l'établissement, qu'ils essaient de tirer le mieux possible leur épingle du jeu sur le marché de l'éducation, qu'ils aient un droit de regard sur la façon dont l'enseignement est dispensé (pratique des enseignants, performances de l'établissement), qu'ils puissent demander des comptes, a contribué à ce que les parents anglais recourent à des pratiques ultra compétitives, et ce sont ceux de la classe moyenne blanche qui réussissent le mieux. Après tout, le néo-libéralisme sert les intérêts de l'individu, en se fondant sur son esprit d'initiative. Comme le montrent Butler et Hamnett (2012), les parents de la classe moyenne ont souscrit à la promesse néo-libérale et à l'importance de la méritocratie. C'est pourquoi, selon ces chercheurs, les parents se retrouvent en concurrence et la responsabilité de l'échec scolaire est attribuée à certaines catégories sociales défavorisées. C'est cette perception des choses qui a conduit les parents de la classe moyenne blanche à convoiter des établissements non fréquentés par les enfants de la communauté noire ou par ceux issus de minorités ethniques ou de catégories socio-économiques défavorisées (Reay et al., 2011). Même les parents de la classe moyenne qui, dans l'étude menée par Reay et al., ont choisi d'envoyer leurs enfants dans la comprehensive school ${ }^{3}$ de leur zone urbaine, sans prendre

3. Comprehensive school : créées dans les années 1960 par le gouvernement travailliste de l'époque, les comprehensive schools sont des établissements polyvalents d'enseignement secondaire conçus pour accueillir tous les élèves sans distinction et leur offrir des chances égales, par opposition au système sélectif des grammar schools. $(N d T)$ 
en compte les performances de l'établissement, ont toutefois veillé à satisfaire leurs intérêts propres : ils ont réussi à obtenir les meilleures ressources, plaçant par exemple leurs enfants dans les meilleures classes ou dans les classes relevant d'un programme spécial. Ainsi, pour les parents dotés d'un plus fort pouvoir d'action, le néo-libéralisme confère un certain nombre d'avantages. Plus récemment, en Angleterre, nous avons pu constater que les demandes des parents de la classe moyenne, visant à obtenir davantage d'établissements, de " meilleure » qualité, ont abouti à la création par le gouvernement de free schools, ou établissements "libres", que les parents peuvent en théorie eux-mêmes mettre en place (avec le soutien de l'État) ${ }^{4}$.

Peu de voix se sont élevées pour dénoncer les inégalités propres à ces pratiques. Reay et al. (2011) ont révélé que, parmi les parents ayant participé à leur enquête, seule une minorité d'entre eux s'est rassemblée et a tenté d'intervenir pour provoquer des changements au sein de l'école et modifier les programmes scolaires. Si les parents de la classe moyenne ont souvent reconnu que les gens sont désavantagés par ces pratiques, peu d'entre eux ont toutefois semblé suffisamment motivés pour changer les choses, ou les remettre en cause. Aux États-Unis, Horvat et al. (2003) ont montré les différences en termes de pouvoir et de ressources des parents de milieux populaires ou de la classe moyenne, pour résister à la politique éducative en place ou aux pratiques qui ont cours dans ce domaine. Ils ont par exemple constaté que les parents de milieux populaires ne bénéficiaient pas de réseaux sociaux leur permettant de se mobiliser pour exprimer leur opposition, contrairement aux parents de la classe moyenne. En Grande Bretagne, il existe effectivement des associations de parents d'élèves à l'intérieur des établissements scolaires, mais leurs pouvoirs ont tendance à être limités. Ces associations s'intéressent davantage à l'organisation d'événements socioculturels ou à la collecte de fonds qu'à toute forme d'activité politique ou d'action de mobilisation. L'enquête réalisée par Vincent (2000) sur les parent-centred organisations (PCO), les associations de défense des intérêts des parents, était au départ fondée sur l'hypothèse que ces associations constituaient des "enclaves de protection" au sein desquelles les parents pouvaient se rencontrer, échanger et mettre en place des réseaux pour mobiliser d'autres parents et faire changer les choses. Certes, cela a bien eu lieu, mais C. Vincent a pu également constater, comme nous l'avons indiqué plus haut, que les questions de classe sociale, de sexe et d'ethnicité affectaient de manière différenciée le degré et le mode de participation et d'investissement des parents.

De façon peut-être plus positive, une étude réalisée par Cork (2005) portant sur cinq associations créées pour venir en aide aux parents de la communauté afro-caribéenne britannique, afin d'améliorer la scolarité et les résultats scolaires de leurs enfants, démontre l'engagement de ces parents et l'énergie

4. Voir Lilistone D., « Angleterre : la "révolution éducative” des academies ", Revue internationale d'éducation de Sèvres, ${ }^{\circ} 60$, p. 15-18. (NdIR) 
qu'ils déploient pour obtenir que le système change et s'améliore. Ces initiatives sont néanmoins peu étendues à l'échelle nationale et demeurent très locales. Elles peuvent dépendre de financements octroyés par l'autorité locale chargée de l'enseignement (financements qui, dans le climat actuel, vont être revus à la baisse), ou être le fruit d'actions menées par des associations bénévoles. Dans un cas comme dans l'autre, elles restent très vulnérables.

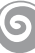

Nous avons donc avancé l'idée que le marché de l'éducation néolibérale a eu un impact profond sur les relations qu'entretiennent les parents avec l'école et sur l'investissement de ces derniers. Leur rôle s'est modifié : d'abord rôle de soutien, puis de client et aujourd'hui de consommateur. Ce processus, que l'on observe depuis de nombreuses années, est solidement établi, et son influence est hégémonique. Comme l'a montré Brown, que nous avons mentionné au début de l'article, le problème central repose sur le caractère inégal des possibilités offertes de s'investir dans ce rôle. Inégal en termes de classe sociale et d'appartenance racial/ethnique. S'il existe quelques exemples de mobilisation parentale et de remise en cause du statu quo, le néo-libéralisme n'a, de manière générale, pas permis d'accroître les chances de réussite pour tous ni d'améliorer le niveau général, et a plutôt eu un effet contre-productif. Les parents n'ont guère d'autre choix que de s'investir dans leur rôle de consommateur, même lorsqu'ils n'ont pas réellement le pouvoir de le faire. L'une des conséquences de cet état de fait est la catégorisation des établissements en " bons » ou " mauvais » et la répartition inégale des ressources.

Ce sont les parents des catégories les plus favorisées qui détiennent le pouvoir, et ce pouvoir est rarement utilisé pour le bien collectif. Les parents ont, semble-t-il, été manipulés et contraints d'endosser un rôle qui exacerbe les inégalités et peut entraîner une certaine disharmonie. La concurrence renforce les intérêts personnels et l'individualisme, alors que la société aurait sans doute besoin de consolider ses valeurs de citoyenneté, de coopération et d'investissement pour le bien collectif.

\section{BibliographiE}

ARCHER L. (2010) 'We raised it with the Head': the educational practices of minority ethnic middle-class families. British Journal of Sociology of Education. 31(4): 449-469. BROWN P. (1990): The 'Third Wave': Education and the Ideology of Parentocracy. British Journal of Sociology of Education. 11(3): 65-85.

BUTLER T. \& HAMNETT C. (2012) : Ethnicity, Class and Aspiration. Bristol: The Policy Press.

CORK L. (2005): Supporting Black Pupils and Parents. London \& New York: Routledge. 
CROZIER G. (2000) : Parents and Schools: partners or protagonists?. Stoke on Trent : Trentham Books.

CROZIER G. \& DAVIES J. (2007) : Hard to reach parents or hard to reach schools? A discussion of home-school relations with particular reference to Bangladeshi and Pakistani parents. British Education Research Journal. 33(3): 295-313.

FOUCAUlT M. (1977) : Discipline and Punish. London : Penguin.

HORVAT E., WEININGER E.B. \& LAREAU A. (2003) : From Social Ties to Social Capital: class differences in the relations between schools and parent networks. American Educational Research Journal. 40(2): 319-351.

LEVITAS R. (1998) : The Inclusive Society. Hampshire and London : Macmillan Press.

REAY D. and BALL S.J. (1998) : 'Making their minds up': family dynamics of school choice. British Educational Research Journal. 24(4): 43-448.

REAY D. CROZIER G. \& JAMES D. (2011) : White Middle-class Identities and Urban Schooling. Hampshire : PalgraveMacmillan.

VINCENT C. (1996) : Parents and Teachers: Power and Participation. London : Falmer Press.

VINCENT C. (2000) : Including Parents? Buckingham and Philadelphia: Open University Press.

WRIGHT C. (1992) : Race Relations in the Primary School. London : David Fulton.

YOUNG I. M. (1990) : Justice and the Politics of Difference. New Jersey, USA : Princetown University Press. 